\title{
Comparison of numerical schemes for computational simulation of liquid wavy film flow on vertical wall
}

\author{
J. K. $\operatorname{Min}^{1}$ \& I. S. Park ${ }^{2}$ \\ ${ }^{1}$ Rolls-Royce University Technology Center, \\ Pusan National University, Korea \\ ${ }^{2}$ School of Mechanical Engineering, \\ Kyungpook National University, Korea
}

\begin{abstract}
Film flows are classified into no-wavy laminar, wavy laminar and turbulence along the Reynolds number or the flow stability. Since the wavy motions of the film flows are so intricate and nonlinear, the studies have largely been dependent upon the experimental way. The numerical approaches have been limited on the no-wavy flow regime. In this study, the film's full wavy motion is numerically investigated. The present numerical approach is based on the VOF interface capturing methods. The implicit and explicit schemes for the interface time marching are compared with each other. The HRIC (High Resolution Interface Capturing) and the geometry reconstruction method are tested as the treatment of convective terms at the liquid-air interface. The dependency of the grid density and the time step size are investigated in the aspect of the probability density function of the film thickness. All results are compared with Takamasa's experimental results.
\end{abstract}

Keywords: wavy film flow, free surface, VOF.

\section{Introduction}

The film flows are generally applied to the phase-change heat transfer (condensation or evaporation) because of their advantages for heat transfer like the high latent heat, the thin film thickness and the large heat transfer area. 
Many previous studies [1-5] proved that the film thickness is the most essential parameter governing heat transfer. Thus a lot of researchers have attempted to apply more reasonable film thicknesses for their applications. Nusselt suggested the exact analytic solution for the film flows in a laminar governing equation set. It is still considered as a very powerful and useful result for simple application problems. Some researchers [6-8] have solved only the convective and diffusive heat transfer equation with the film thickness and velocity distribution from the Nusselt solution as the flow information. They couldn't be applied to the accurately predicting the local heat and mass transfer in the developing region. Thereafter a lot of researchers [3-5] attempted to numerically solve the parabolized Navier-Stokes equation as the governing equation with the marching technique. This has been evaluated as the more accurate numerical approach by considering the convective heat transfer effect by the normal velocity component which has been neglected in the thin film flow so far. Because the parabolized governing equations were solved, this method was applicable to the laminar as well as the turbulence flow regimes and found the average film thickness reasonably. But it could not solve the time changing phenomena such as the film's wavy motion which happened due to the flow instability in higher flow rate conditions.

There have been attempts to solve the elliptic type full Navier-Stokes equations [9-12]. Since this method could additionally consider the diffusion in the primary flow direction, it was advantageous for the falling film on the horizontal circular tube. The numerical free surface capturing or tracking methods such as MAC (Marker and Cell) [13], VOF (Volume Of Fluid) [14], moving grid technique [15] and so on, were applied to investigate the film's free surface location precisely.

The analysis for the three dimensional film flows with secondary flows in the cross section like the grooved, fluted or spiral tube was conducted [11, 12]. The approaches using the elliptic governing equations which were investigated with a flourish in the late 1990s could make no more evolutions because of their weakness of high computational cost comparing with the advantages from their accurate calculation.

It is known that the film flow is getting unstable as the flow rate increases. Thus the laminar wavy motion starts to appear over Reynolds number 30 and the flow transits to the turbulence between Reynolds 1,600 and 3,200. Most applications belonged in the laminar wavy regime to apply the heat-transferboosting effects by the interfacial wave and the thin film thickness. Since waves in the turbulence regime are highly frequent and short waves, the heat and mass transfer enhancement by the wavy motion can be somewhat simulated by adjusting the turbulence intensity. But the waves of long wavelength and high amplitude in the low Reynolds number need the wavy motion itself to be accurately solved. Because the wavy motion of film flow means the temporary and spatially fluctuating film thickness, its height (amplitude), frequency, and velocity are as important as the average film thickness.

Complex interactions between the liquid turbulence structure in the film and gas-liquid interfaces govern the physical properties of such flows. The resulting 
interfacial waves show fascinating nonlinear phenomena, including solitary waves and complex disordered patterns. To elucidate these phenomena in detail, spatial-temporal knowledge of the interfacial waves is essential.

Due to the limitation of the numerical approaches, the experimental techniques measuring film flow such as electrical-resistance or electricalcapacitance methods have widely developed [16-22]. The electrical methods can measure the film's spatially averaged thickness, but not the film's wavy behaviors (wave amplitude, frequency, and so on). A laser displacement sensor [21] and a supersonic echo method [22] have a higher spatial resolution and thus it becomes possible to measure the film's wavy characteristics. But for the cases of large curvature gas-liquid interface, the measuring is impossible because the reflected laser beam and the sonic wave cannot reach the detector. Takamasa and Hazuku [23] successfully measured the wavy characteristics of the films flowing down a vertical plate wall and a vertical tube wall with a sensitivity of $2 \mu \mathrm{m}$ and $1 \mathrm{kHz}$. They used the laser focus displacement method with the high spatial and temporal resolution. A lot of studies have been conducted with the above experimental ways to reveal not only the film's wavy motion itself (such as wave celerity, amplitude and frequency) but also the relationship with the heat transfer.

In this study, various numerical schemes are tested for the laminar wavy film flow region. The present numerical approach is based on the VOF interface capturing methods. The implicit and explicit schemes for the interface time matching are compared with each other. The HRIC (High Resolution Interface Capturing) and the geometry reconstruction method are tested as the treatment of convective terms at the liquid-air interface. The dependency of the grid density and the time step size are investigated in the aspect of the probability density function of the film thickness. All results are compared with Takamasa's experimental results [23]. In addition the results obtained from turbulence flow simulation are compared together.

\section{Numerical analysis}

We consider a vertical wall on which the wavy laminar film flows as shown in Fig. 1. For constant fluid properties, the continuity and momentum equations for two-dimensional incompressible unsteady laminar film flows are written as:

$$
\begin{gathered}
\frac{\partial}{\partial x_{i}}\left(\rho u_{i}\right)=0 \\
\frac{\partial\left(\rho u_{i}\right)}{\partial t}+\frac{\partial}{\partial x_{j}}\left(\rho u_{i} u_{j}\right)=-\frac{\partial p}{\partial x_{j}}+\frac{\partial}{\partial x_{j}}\left[\mu\left(\frac{\partial u_{i}}{\partial x_{j}}+\frac{\partial u_{j}}{\partial x_{i}}\right)\right]+\rho g_{i}
\end{gathered}
$$

where, $u_{i}$ is the velocity vector, $p$ pressure, $g_{i}$ gravity vector, $\rho$ density and $\mu$ viscosity of the fluid. To describe the motion of free surface, the VOF (Volume Of Fluid) approach adopts the volume fraction function $\alpha$ which has a value between zero to unity according to the fraction of liquid inside each control volume. The corresponding equation for the volume fraction function is: 


$$
\frac{\partial \alpha}{\partial t}+\frac{\partial}{\partial x_{j}}\left(\alpha u_{j}\right)=0 .
$$

Using this volume fraction $\alpha$, the effective material properties in Eqs. (1) and (2) can be evaluated as follows:

$$
\begin{aligned}
& \rho=\alpha \rho_{l}+(1-\alpha) \rho_{g} \\
& \mu=\alpha \mu_{l}+(1-\alpha) \mu_{g}
\end{aligned}
$$

where the subscripts $l$ and $g$ represents the liquid and gaseous phases, respectively.

The boundary conditions for this problem are also shown in Fig. 1. On the liquid inlet boundary, the uniform velocity $u_{0}$ and fixed film thickness $\delta_{0}$ are specified according to the corresponding Reynolds numbers. No-slip condition is applied on the vertical wall and gas inlet. Pressure outlet conditions are used for the other boundaries. On the free surface, the surface tension force is taken into consideration to satisfy the following force balance equation:

$$
p_{l}-p_{g}=\sigma \frac{1}{R}
$$

where $\sigma$ represents the surface tension coefficient and $R$ the radius of curvature of the free surface. In the numerical procedure, the CSF (Continuum Surface Force) model by Brackbill et al. [24] has been used. In this method, the surface curvature $\kappa$ can be evaluated by the divergence of surface normal which is defined as the normalized gradient of volume fraction $\alpha$. To impose the pressure jump across the surface, the volume force defined by the following equation should be applied for the control volume at the free surface.

$$
F_{v o l}=\sigma \frac{\rho \kappa \nabla \alpha}{\frac{1}{2}\left(\rho_{l}+\rho_{g}\right)}
$$

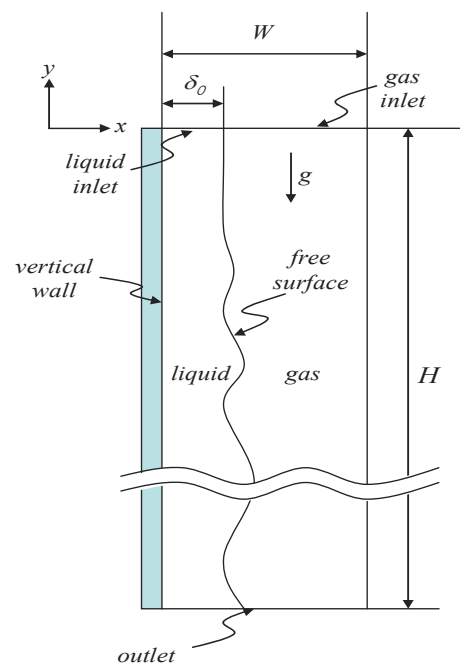

Figure 1: $\quad$ Schematic of wavy film flow. 
For the numerical computations, the commercial CFD code FLUENT 6.3 has been used. Second order upwind scheme for the spatial discretization, implicit time marching and explicit time discretization for the VOF equation were adopted. In order to capture the location of interface precisely, the volume fraction $\alpha$ should vary sharply near the free surface of the liquid. VOF approach has the possibility of smearing the fraction function due to the false diffusion of numerical scheme. This problem is largely related to the numerical evaluation of flux values at the control surface of partially filled cell near the interface. Numerous computational techniques to improve the accuracy of surface tracking by VOF have been studied. Hirt and Nichols [14] suggested the donor and acceptor method in their initial VOF technique. According to this method, a cell near the free surface can be identified as a donor and the neighbouring cell as the acceptor depending on the flow direction at the control surface. The amount of the fluid from each phase across the control surface is calculated using the volume fraction value at the donor cell and the acceptor cell is assumed to receive the same amount of fluid from the donor cell. This method also determines the orientation of free surface using the gradient value of volume fraction $\alpha$ at the cell near the free surface, which can be either horizontal or vertical. Using the determined orientation and the velocity of the interface, the flux values across the control surface can be evaluated by modified upwinding technique in order to inhibit the false diffusion. Youngs [25] has suggested the PLIC (piecewise linear interface calculation) technique. In this method, the slope of the interface at the cell can be evaluated using the volume fraction values of surrounding neighboring cells. With this linear slope and the motion of the interface, the fluid flux across the control surface can be calculated as well.

Water is used for the liquid film flow and air for the gaseous phase in the present study. The width of liquid film $\delta_{0}$ at the inlet is set to $0.4-0.6 \mathrm{~mm}$ based on average film thickness, the height of the domain $H=500 \mathrm{~mm}$ and the width $W=4.6 \mathrm{~mm}$. Corresponding dimensionless parameters are defined as follows:

$$
\begin{aligned}
R e & =\frac{\rho u_{0} \delta_{0}}{\mu} \\
W e & =\frac{\rho \delta_{0} u_{0}^{2}}{\sigma}
\end{aligned}
$$

where $R e$ is the Reynolds number and $W e$ the Weber number. The Reynolds number has the range of 200 to 1000 and the inlet velocity for each $R e$ can be calculated using the definition, eq. (8).

A $40 \times 3000$ grid was generated for the computational domain, which was clustered to the wall and uniform in the flowing direction. Several test showed that the Courant number $\operatorname{Co}=\Delta t /\left(\Delta x_{\text {cell }} / v_{\text {fluid }}\right)$ should be less than 0.5 in order to get a stable solution. As a result, the time step was selected to have $\Delta t=10^{-4} \mathrm{sec}$, which showed $C o=0.43$ for $R e=1000$ case. For the computation, machines having Intel Xeon processors with eight nodes were used and it took 10-24 hours of CPU time to get a solution for 15000 time steps. 


\section{Results and discussions}

For the discretization scheme at the cell near the free surface, the HRIC (High Resolution Interface Capturing) scheme is known as an improved scheme providing better accuracy. This is a blending scheme of upwind and downwind differencing. In this study, however, the use of HRIC scheme produced less realistic results. Table 1 shows the cases for testing the numerical schemes for the time marching and the interfacial cell treatment. Fig. 2 shows the distributions of the probability density function for the film thickness for each case. Comparing with Takamasa's experimental result, the explicit time marching and geometry reconstruction method is best fit with.

Fig. 3 shows the time variations of film thickness calculated using explicit HRIC scheme, implicit HRIC scheme and geometric reconstruction scheme, respectively. Geometric reconstruction scheme is a generalized Youngs' method and used with second-order discretization scheme in this calculation. It is hard to identify a solitary wave when explicit and implicit HRIC schemes were used, whereas a distinct solitary wave is observed with geometric reconstruction scheme, which is closer to previous experimental findings [23].

For the comparison of the effects by the grid and time step sizes, two grid systems (grid spacing $=0.5$ and $0.2 \mathrm{~mm}$ ) and three time step sizes $\left(2 \times 10^{-4}, 1 \times 10^{-4}\right.$, and $0.5 \times 10^{-4}$ sec. $)$ are tested. Fig. 4 shows the distributions of the probability density functions for each case. For the cases of grid size, 0.5 $\mathrm{mm}$, much wider ranges of film thickness are predicted than the cases of grid size, $0.2 \mathrm{~mm}$. As the time step sizes become smaller, the distribution becomes sharper. But the gap with the experimental result is narrowed no more. The average film thickness is about $0.5 \mathrm{~mm}$ as shown in the probability density function. This means that finer grid spacing than the average film thickness is required for the accurate calculation. The results of grid size $0.2 \mathrm{~mm}$ are more realistic comparing with Takamasa's experimental data and invariant with the time step size.

The film Reynolds number handled in this study is ranged between 200 and 1,000 . This is known as the laminar wavy film flow region. However, the wavy motion is a transient phenomenon to the turbulence, and the wave itself is a large fluctuation and originated by the disturbance from outside. Such generated waves are amplified and sometimes damped out according to the mass flow rate. In this study, to investigate the film flow's turbulence effect, we adopt the two

Table 1: $\quad$ Test cases for inspecting numerical scheme for time differencing and interfacial cell treatment.

\begin{tabular}{c|c|c|c}
\hline Case & Time Differencing & VOF & Viscosity \\
\hline 1 & Explicit & Geometry Reconstruction & Laminar \\
\hline 2 & Explicit & HRIC & Laminar \\
\hline 3 & Implicit & HRIC & Laminar \\
\hline
\end{tabular}




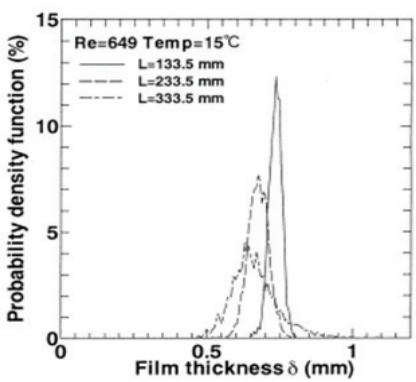

(a)

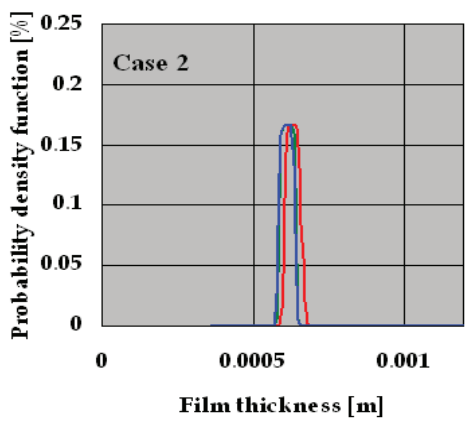

(c)

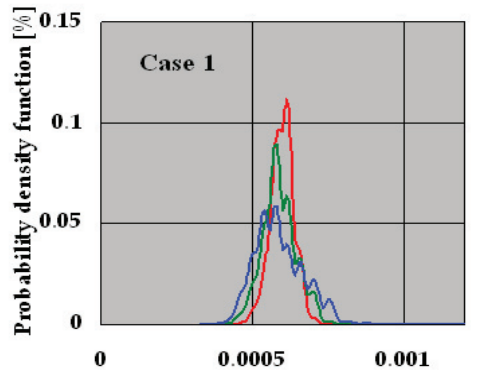

Film thickness [m]

(b)

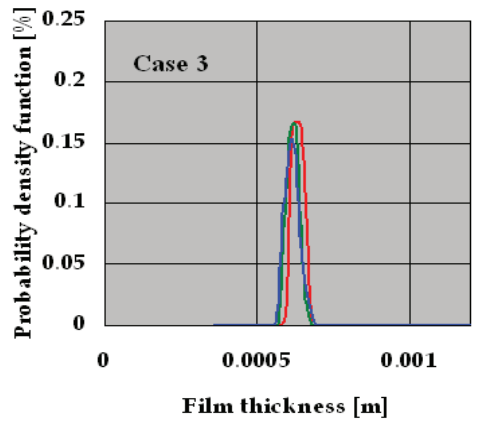

(d)

Figure 2: Probability density functions for film thickness at $R e=649$, (a) Takamasa's experiment, (b) Explicit geometry reconstruction, (c) Explicit HRIC, and (d) Implicit HRIC.

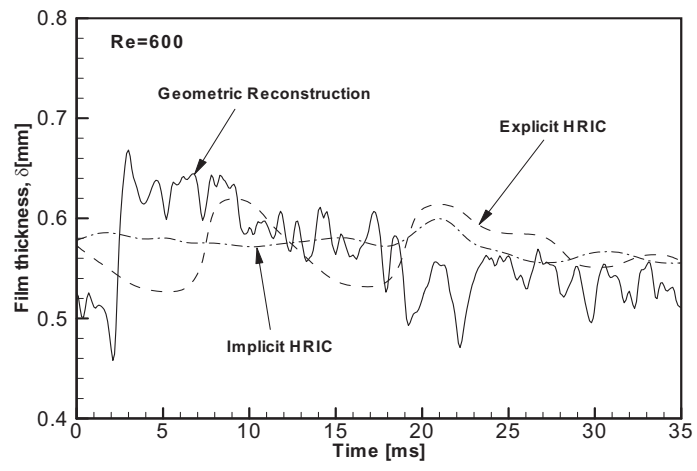

Figure 3: Comparison of interface cell treatment-geometry reconstruction scheme and HRIC scheme. 

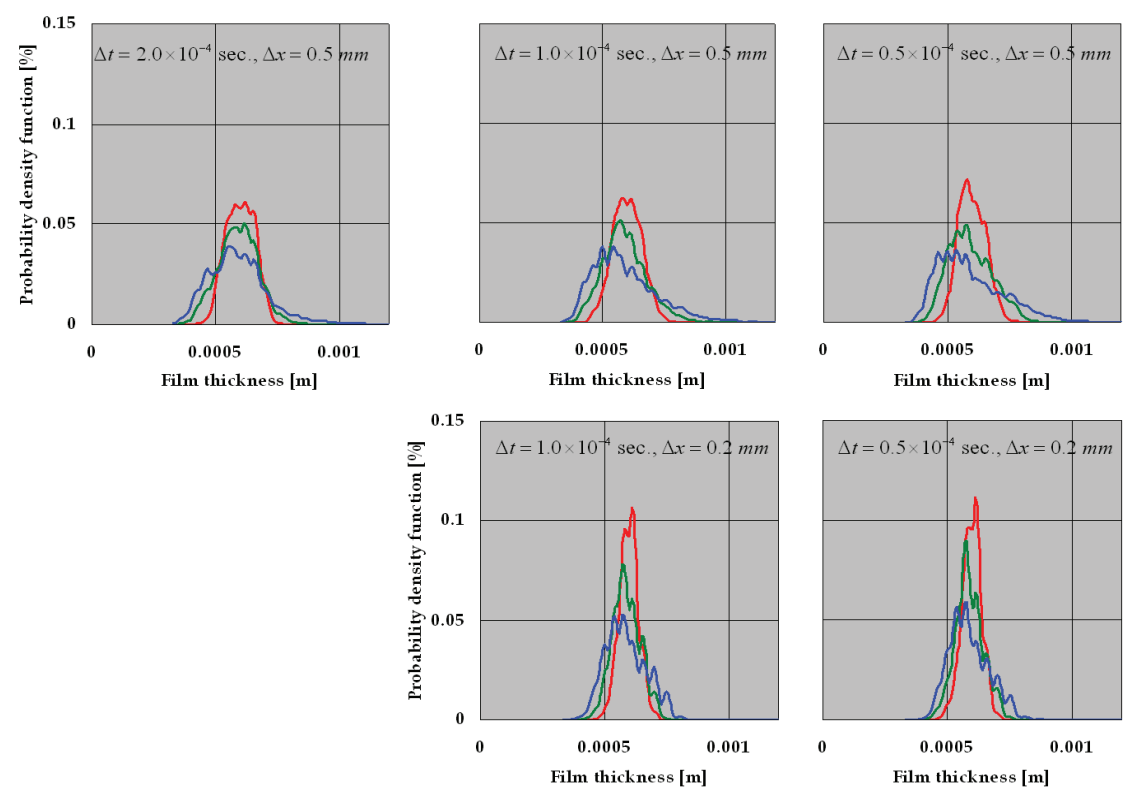

Figure 4: Probability density functions for various time step and grid sizes at $\mathrm{Re}=649$.

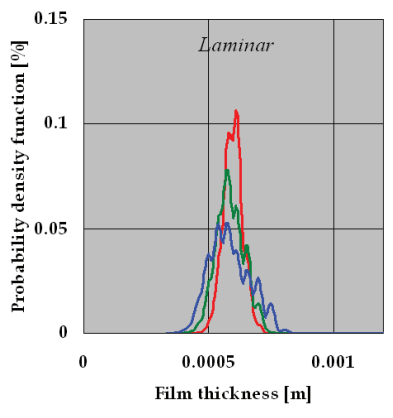

(a)

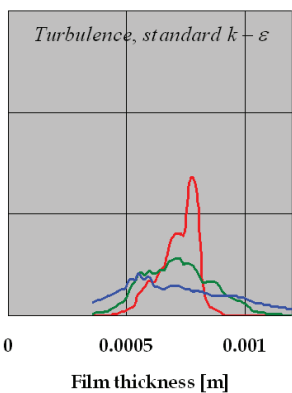

(b)

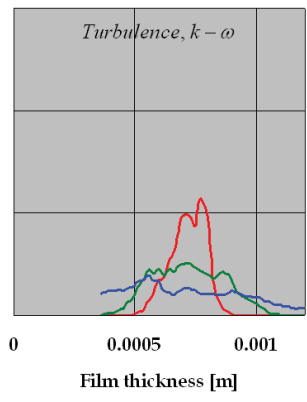

(c)

Figure 5: Comparison with turbulence simulations at $\mathrm{Re}=649$, (a) Laminar, (b) Standard $k-\varepsilon$, and (c) $k-\omega$ turbulence model.

generalized 2-equation turbulence models (standard $k-\varepsilon$ model and $k-\omega$ model) and compared the results with the laminar simulation. Fig. 5 compares the results one another. The Reynolds number of test case is 649. The turbulence simulations generate more severe wave fluctuations because of the turbulence fluctuation. Both turbulence analyses show a quite different wavy motions comparing with the real case. Thus in the present Reynolds number range (200$1,000)$, when the flow is simulated by the laminar assumption, more reasonable results can be obtained. 
Fig. 6 shows the wave's time-propagation processes for Reynolds number 200. To accurately observe and compare the wave shapes, the figures are made with $\mathrm{x}-\mathrm{y}$ aspect ratio 5:1. The solitary waves with high wave amplitude appear and the foregoing small waves are merged at several points by the big wave approaching from upstream. The small amplitude ripples are observed among the solitary waves.
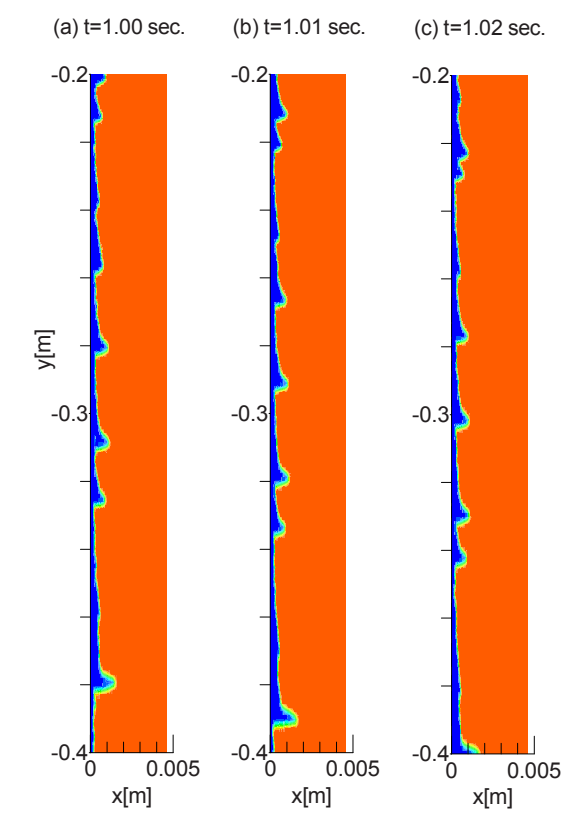

(d) $\mathrm{t}=1.03 \mathrm{sec}$.

(e) $\mathrm{t}=1.04 \mathrm{sec}$.

(f) $\mathrm{t}=1.05 \mathrm{sec}$.
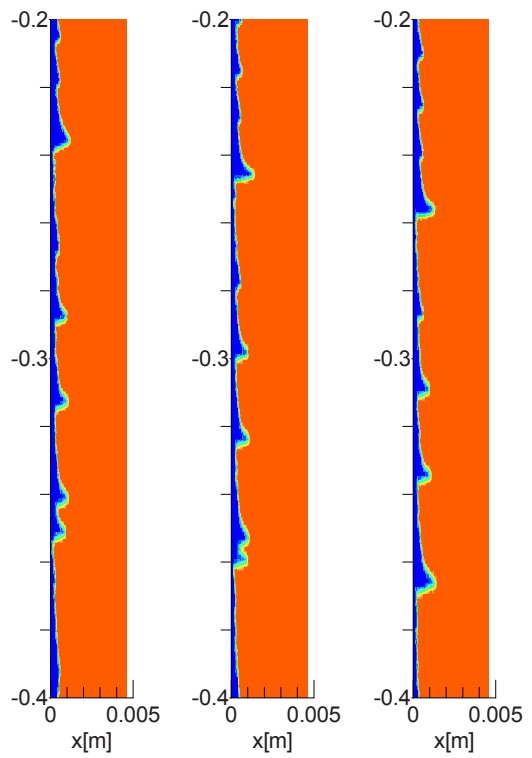

Figure 6: Propagation of wave along time at $\mathrm{Re}=200$.

Fig. 7 shows the relationship between average film thickness and the Reynolds number. The average film thickness is defined as the arithmetic mean value of the instantaneous film thicknesses and calculated as follows.

$$
\bar{\delta}=\frac{1}{n} \sum_{i=1}^{n} \delta_{i}
$$

The Nusselt's theoretical result for laminar flow condition is

$$
\bar{\delta}=\left(\frac{3 v^{2} R e}{g}\right)^{1 / 3}
$$

The universal velocity profile is Karman's velocity profile (Eq. (12)) calculated for turbulent flow condition.

where

$$
\left(3.0+2.5 \ln \bar{\delta}^{+}\right) \bar{\delta}^{+}=\operatorname{Re}+64
$$


38 Computational Methods in Multiphase Flow VI

$$
\begin{aligned}
& \bar{\delta}^{+}=u^{*} \frac{\bar{\delta}}{v} \\
& u^{*}=\sqrt{g \bar{\delta}}
\end{aligned}
$$

The results by Brauer (Eq. (15)), Takahama (Eq. (16)), and Aragaki (Eq. (17) shown in the figure are represented as follows:

$$
\begin{gathered}
\bar{\delta}=0.302\left(\frac{3 v^{2}}{g}\right)^{1 / 3} R e^{0.526} \\
\bar{\delta}=0.473\left(\frac{v^{2}}{g}\right)^{1 / 3} R e^{0.526} \\
\bar{\delta}=\left(\frac{v^{2}}{g}\right)^{1 / 3}\left[8.92 R e^{5 / 2}+4.04 \times 10^{-5} R e^{9 / 2}\right]^{2 / 15}
\end{gathered}
$$

Takamasa's result is the experimental one obtained by their laser focus displacement method. The present results agree well with Nusselt and Takamasa's results especially in the far downstream region.

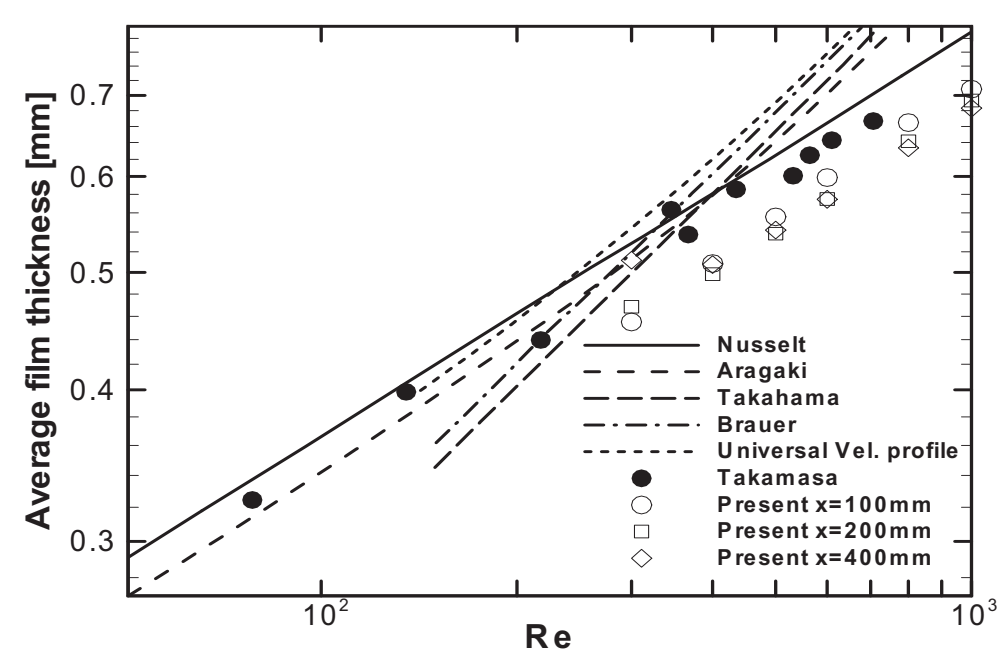

Figure 7: Average film thickness for various Reynolds numbers and various positions from liquid inlet.

\section{Conclusions}

For a film flow heat transfer which is widely applied due to its benefits for heat transfer. The film flow becomes unstable with the wave propagation over the Reynolds number 30. In this study, the wavy motions of the film flows were numerically investigated, which have largely depended on the experimental ways. The interfaces between liquid and gas were sharply captured by the VOF 
and PLIC method. The surface tension acting on the interfaces was correctly calculated from a well defined interface shape by the VOF and PLIC. Such correctly estimated surface tension forces were able to propagate waves on the interface correctly. The present numerical results showed a good agreement with the experimental one in the aspects of the average film thickness. It is clearly demonstrated that the current numerical Navier-Stokes procedure and precise free-surface capturing method with consideration of surface-tension effect are essential in order to predict the wavy film motion accurately.

\section{Acknowledgement}

This research was supported by Basic Science Research Program through the National Research Foundation of Korea (NRF) funded by the Ministry of Education, Science and Technology (the Grant 2010-0024619).

\section{References}

[1] Moon-Hyun Chun \& Kyun-Tae Kim, Assessment of the new and existing correlations for laminar and turbulent film condensations on a vertical surface, Int. Commun. Heat Mass Transfer, 17, pp. 431-441, 1990.

[2] A. Faghri \& R, A. Seban, Heat transfer in wavy liquid films, Int. J. Heat Mass Transfer, 28 (2), pp. 506-508, 1985.

[3] M. Feddaoui, A. Mir \& E. Belahmidi, Cocurrent turbulent mixed convection heat and mass transfer in falling film of water inside a vertical heated tube, Int. J. Heat Mass Transfer, 46, pp. 3497-3509, 2003.

[4] M. K. Groff, S. J. Ormiston \& H. M. Soliman, Numerical solution of film condensation from turbulent flow of vapor-gas mixtures in vertical tubes, Int. J. Heat Mass Transfer, 50, pp. 3899-3912, 2007.

[5] I. S. Park \& M. Y. Kim, Numerical investigation of the heat and mass transfer in a vertical tube evaporator with the three-zone analysis, Int. J. Heat Mass Transfer, 52, pp. 2599-2606, 2009.

[6] N.I. Grigoreva \& V.E. Nakoryakov, Exact solution combined heat and mass transfer problem during film absorption, Journal of Engineering Physics, 33(5), pp. 1349-1353, 1977.

[7] G. Grossman, Simultaneous heat and mass transfer in film absorption under laminar flow, Int. J. Heat Mass Transfer, 25 (3), pp. 357-371, 1982 .

[8] G. Grossman \& M.T. Heath, Simultaneous heat and mass transfer in film absorption in turbulent liquid films, Int. J. Heat Mass Transfer, 27(12), pp. 2365-2376, 1984.

[9] E. Stuhlträger, Y. Naridomi, A. Miyara \& H. Uehara, Flow dynamics and heat transfer of a condensate film on a vertical wall-i. Numerical Analysis and Flow Dynamics, Int. J. Heat Mass Transfer, 36, pp. 1677-1686, 1993.

[10] J.K. Min \& D.H. Choi, Analysis of the absorption process on a horizontal tube using Navier-Stokes equations with surface-tension effects, Int. J. Heat Mass Transfer, 42 (24), pp. 4567-4578, 1999. 
[11] I.S. Park \& D.H. Choi, Heat- and mass-transfer analysis for the condensing film flow along a vertical grooved tube, Int. J. Heat Mass Transfer, 44(22), pp. 4277-4285, 2001.

[12] I.S. Park, Numerical analysis for flow, heat and mass transfer in film flow along a vertical fluted tube, Int. J. Heat Mass Transfer, 53(1-3), pp. 309-319, 2010.

[13] F.H. Harlow \& J.E. Welch, Numerical calculation of time-dependent viscous incompressible flow of fluid with free surface, Physics of Fluids, 8 pp. 2182-2189, 1965.

[14] C.W. Hirt \& B.D. Nichols, Volume of fluid (vof) method for the dynamics of free boundaries, Journal of Computational Physics, 39, pp. 201-225, 1981.

[15] S. Muzaferija \& M. Peric, Computation of free-surface flows using the finite-volume method and moving grids, Numerical Heat Transfer, Part, $B, 32$, pp. 369-384, 1997.

[16] S. Poltalski \& A.J. Clegg, An experimental study of wave inception on falling liquid film, Chemical Engineering Science, 27, pp. 1257-1265, 1972.

[17] K. Mori, T. Matsumoto \& H. Uematsu, Time-spatial interfacial structures and flow characteristics in falling liquid film, Proceedings of Third International Conference on Multiphase Flow, CD-ROM, \#514, 1998.

[18] A. Elsäßer, W. Samenfink, J. Ebner, K. Dullenkopf \& S. Wittig, Effect of variable liquid properties on the flow structure within, Proceedings of Ninth International Symposium on Application of Laser Techniques to Fluid Mechanics, 1(3), pp. 1-10, 1998.

[19] A.E. Dukler \& O.P. Bergelin, Characteristics of flow in falling liquid film, Proceeding of Chemical Engineering Progress Symposium Series, 48(11), pp. 557-563, 1952.

[20] C.F. Hewitt \& N.S. Hall-Taylor, Annular Two-phase Flow, Pergamon Press, Oxford, 1970.

[21] M. Nasr-Esfahany \& M. Kawaji, Turbulence structure under a typical shear induced wave at a liquid/gas interface, Proceedings of AIChE Symposium Series, 310(92), pp. 203-210, 1996.

[22] A. Serizawa, T. Kamei, I. Kataoka, Z. Kawara, T. Ebisu \& K. Torikoshi, Measurement of dynamic behavior of a liquid film flow with liquid droplets in a horizontal channel, Proceedings of Second International Conference on Multiphase Flow, 2, pp. 27-34, 1995.

[23] T. Takamasa \& T. Hazuku, Measuring interfacial waves on film flowing down a vertical plate wall in the entry region using laser focus displacement meters, Int. J. Heat Mass Transfer, 43, pp. 2807-2819, 2000.

[24] J.U. Brackbill, D.B. Kothe \& C. Zemach, A continuum method for modeling surface tension, Journal of Computational Physics, 100, pp. 335-354, 1992.

[25] D.L. Youngs, Time-dependent multi-material flow with large fluid distortion, numerical methods for fluid dynamics, Academic Press, pp. 273-285, 1982. 